\title{
Valoración de opciones reales con múltiples incertidumbres mediante modelos k-dimensionales
}

\author{
Real Options valuation with multiple uncertainties \\ using k-dimensional models
}

Carlos Andrés Zapata Quimbayo*

* $\quad$ Magíster en Finanzas. Docente-Investigador, Observatorio de Economía y Operaciones Numéricas (ODEON) de la Universidad Externado de Colombia. Bogotá (Colombia).

[carlosa.zapata@uexternado.edu.co].[https://orcid.org/0000-0003-3337-0182].

Fecha de recepción: 1 de febrero de 2019.

Fecha de aceptación: 4 de abril de 2019.

Para citar este artículo:

Zapata Quimbayo, C. A. (2019). Valoración de opciones reales con múltiples incertidumbres mediante modelos $k$-dimensionales. ODEON, 16, pp. 97-121.

DOI: https://doi.org/10.18601/17941113.n16.05 


\section{Resumen}

El modelo binomial presenta un conjunto de propiedades que lo convierten en el enfoque adecuado para resolver el problema de valoración en el campo de las opciones reales, mediante una aplicación fácil y práctica. Cabe resaltar que esto solo es posible en el contexto donde se presenta una única fuente subyacente de incertidumbre. Sin embargo, su adopción puede ser limitada para aquellas inversiones que cuentan con múltiples incertidumbres, dado que su interacción debería incorporarse al proceso de valoración. Como respuesta, la teoría ha propuesto enfoques de valoración que permiten representar las diferentes fuentes de incertidumbre mediante una estimación consolidada de la volatilidad, tal es el caso del enfoque Marketed Asset Disclaimer (MAD) desarrollado por Copeland y Antikarov (2001). Como alternativa, se puede dar un tratamiento individual a cada incertidumbre. En este contexto, se encuentran diferentes propuestas que extienden el modelo binomial a un contexto k-dimensional, donde se incorpora la dinámica propia de cada incertidumbre, así como sus correlaciones. Para lograr esta aplicación, se requiere una aproximación al proceso estocástico $k$-dimensional. Este trabajo presenta una revisión sucinta de los diferentes métodos propuestos en este contexto, así como sus bondades, limitaciones y algunos enfoques alternativos.

Palabras clave: opciones reales, procesos $k$-dimensionales, valoración.

Clasificación JEL: C63, G12, G13. 


\section{Abstract}

The binomial model presents a set of properties that make it a suitable approach in order to value the real options, throughout an easy and practical application. This is possible by the adaptation of the valuation principle for non-arbitrage, own of the options pricing theory. However, their adoption may be limited for those options that have multiple sources of uncertainty, given that their interaction should be incorporated into the valuation process. In response, financial theory has proposed valuation approaches that allow different sources of uncertainty to be represented by a consolidated estimate of volatility, such as the Marketed Asset Disclaimer (MAD) approach developed by Copeland and Antikarov (2001). As an alternative, a treatment that incorporates the dynamics of each uncertainty can be given. In this context, there are different proposals that extend the Binomial model to k-dimensional or multi-dimensional context. To achieve the application, it is necessary an approximation of the $k$-dimensional stochastic process, as well as its correlations. This paper presents a concise review of the different methods proposed in this context, as well as their benefits, limitations and, some alternative approaches.

Key words: real options, $k$-dimensional process, valuation.

JEL classification: C63, G12, G13. 


\section{Introducción}

En el mundo actual de los negocios, las empresas se enfrentan a múltiples fuentes de incertidumbre cuando toman decisiones sobre futuras oportunidades de inversión. En este sentido, realizar una valoración correcta de estas oportunidades es más complejo de lo que el modelo tradicional de valoración (el flujo de caja descontado - FCD) puede sugerir (Dixit y Pindyck, 1994; Smit y Trigeorgis, 2004), dadas las limitaciones que este presenta por su carácter estático ${ }^{1}$. Sin embargo, un análisis de valoración adecuado requiere que la empresa pueda revisar o adaptar sus decisiones de inversión en respuesta a los acontecimientos futuros que presenta el mercado. Cada decisión de inversión, por ejemplo, puede estar acompañada de estrategias óptimas que permiten tomar cursos alternativos como: expandir cuando se identifiquen mejores condiciones en el mercado; contraer o abandonar cuando el resultado sea peor de lo esperado (Trigeorgis, 1996; Amram y Kulatilaka, 1999), o incluso aplazar la inversión durante un tiempo determinado mientras se resuelve parte de esta incertidumbre. Estas decisiones representan opciones reales que acompañan el proceso de toma de decisión como respuesta a la alta incertidumbre y la flexibilidad estratégica. De esta forma, el análisis de opciones reales (ROA, por su sigla en inglés), permite obtener valoraciones más precisas y realistas (Copeland y Antikarov, 2001) en comparación con el modelo tradicional.

Además, Dixit y Pindyck (1994) y Trigeorgis (1996) reconocen que el modelo FCD presenta una deficiencia notable, producto del abandono total de la naturaleza incierta de los flujos de caja, y de las respuestas estratégicas de la gerencia para su gestión. Una ventaja adicional del ROA es que permite reducir las complejas estructuras corporativas y de los proyectos de inversión a simples estructuras analíticas conformadas por diferentes tipos de opciones reales simples con fácil aplicación (Smit y Trigeorgis, 2004).

Cabe resaltar que la aplicación del ROA ha sido posible gracias a la adaptación de los modelos de valoración de opciones financieras: el modelo Back-ScholesMerton (BSM) (Black y Scholes, 1973; Merton, 1973) y el modelo binomial (Cox, Ross y Rubinstein, 1979). Estos aportes se identifican inicialmente en los trabajos de Merton (1977) ${ }^{2}$ y Myers (1977). Al igual que las opciones financieras, el mode-

${ }^{1}$ El modelo estándar de valoración (FCD) define un criterio rígido para la toma de decisión de inversión basado en el valor presente neto (VPN), es decir, si el VPN $>0$, la inversión se ejecuta, de lo contrario se rechaza. Además, este enfoque asume que la inversión no se puede modificar una vez se lleva a cabo.

${ }^{2}$ Este primer desarrollo se identifica en el análisis de reclamaciones contingentes (CCA). El 
lo de valoración de opciones reales se basa en el supuesto de que el activo subyacente, es decir, el valor presente de los activos de la empresa o el valor presente del proyecto de inversión $(V)$, sigue un proceso estocástico del tipo movimiento geométrico Browniano (MGB) y se requiere como parámetros de entrada: i) el valor actual del proyecto $\left(V_{0}\right)$; ii) el precio de ejercicio ${ }^{3}$; iii) la tasa de interés libre de riesgo $(r)$; iv) el vencimiento de la opción $(T)$; y v) la volatilidad futura del valor del proyecto $(\sigma)$.

De estos parámetros, la volatilidad futura, definida como la desviación estándar de la tasa de rendimiento del valor presente del proyecto, es la única que no puede ser observada directamente y representa un parámetro crítico del modelo de valoración, por lo que su estimación se convierte en un verdadero reto en la aplicación del ROA. Mientras que en una opción financiera se utilizan datos históricos del activo subyacente para estimar la volatilidad, la cual es usada como proxy de la volatilidad futura ${ }^{4}$, para una opción real, según Copeland y Antikarov (2001), su estimación presenta varias dificultades:

- Al tratarse de activos no negociables, los proyectos de inversión carecen de un mercado, no se cuenta con datos históricos para su estimación.

- Un proyecto presenta características propias a nivel técnico, financiero y de gestión, que difícilmente lo vuelve comparable con otros o con el mismo portafolio de mercado. Por tanto, la búsqueda del activo o portafolio perfectamente correlacionado es difícil.

- El valor presente de la inversión depende del comportamiento futuro de múltiples factores o fuentes de incertidumbre. Las principales incertidumbres están relacionadas con el funcionamiento mismo del mercado, la tecnología, los cambios en la regulación, entre otros.

Además, la valoración de opciones financieras se fundamenta en el principio de no arbitraje, en el que el valor de una opción puede ser deducido a través de portafolios de activos comercializados que replican sus pagos en todos los estados de naturaleza. Como resultado, se encuentra una capacidad limitada en su aplicación y,

CCA representa la primera respuesta para la valoración de la empresa a partir del tratamiento de su estructura de capital: deuda, acciones y bonos convertibles, y evalúa cómo cambia el valor de estas reclamaciones y el valor de los activos en el tiempo.

${ }^{3}$ Por ejemplo, en una opción real de invertir este representa el monto de la inversión $(I)$.

${ }^{4}$ De igual forma, al contar con precios de mercado de la opción, se puede inferir la volatilidad al invertir la fórmula de valoración de Black-Sholes-Merton para obtener la volatilidad implícita. 
por tanto, su uso debe ser cuidadoso (Trigeorgis, 1996). Así, un tratamiento adecuado de las opciones reales requiere de nuevos supuestos que permitan superar estas dificultades en el proceso de valoración.

La teoría (convencional) de opciones reales ha realizado un abordaje de esta problemática desde diferentes enfoques (Borison, 2005). Por ejemplo, Copeland y Antikarov (2001) proponen un enfoque consolidado de estimación, conocido como Marketed Asset Disclaimer (MAD), para estimar el efecto conjunto de todas las incertidumbres en una sola estimación fiable de la volatilidad del proyecto de inversión ${ }^{5}$. De esta forma, Copeland y Antikarov (2001) construyen un modelo de estimación que busca capturar la variabilidad de las diferentes fuentes de incertidumbre apoyados en la técnica de simulación de Monte Carlo. Para ello, toman como activo subyacente el valor del proyecto sin opciones (VPN estático), asumiendo que este valor puede representar objetivamente su verdadero valor de mercado ${ }^{6} \mathrm{y}$, de esta forma aceptan que este varía en el tiempo según un proceso estocástico del tipo MGB. Una vez es superado el problema de estimación de la volatilidad, es posible construir un árbol binomial para aproximar la evolución del valor del proyecto en el tiempo usando el modelo de Cox et al. (1979).

El modelo binomial permite superar parte de estas limitaciones, como indican Copeland y Tufano (2004). Por ejemplo, este modelo se puede ajustar fácilmente para reflejar cambios en la volatilidad, los puntos (óptimos) de decisión temprana, las decisiones secuenciales (opciones compuestas), las cuales son comunes en el contexto de las opciones reales. Esta flexibilidad del modelo permite reflejar las características idiosincráticas de los proyectos de inversión utilizando herramientas de fácil manejo, como la hoja de cálculo de Excel. Además, Brandão, Dyer y Hahn

${ }^{5}$ Una aproximación alternativa, bajo este mismo enfoque, se encuentran en Brandão, Dyer y Hahn (2005). Por su parte, se han realizado esfuerzos interesantes para resolver esta dificultad mediante la búsqueda de una solución analítica de la volatilidad. En este campo, se resalta el trabajo de Davis (1998), quien basado en los trabajos de Brennan y Schwartz (1985), Paddock, Siegel y Smith (1988) y Dixit y Pindyck (1994), propone tomar como volatilidad del proyecto la misma de la principal fuente subyacente de incertidumbre. $\mathrm{Al}$ asumir que el precio del producto final del proyecto sigue un proceso de difusión del tipo MBG, Davis (1998) relaciona su variabilidad con la volatilidad total del proyecto, y obtiene así una estimación que se puede representar de forma analítica. Propuestas similares se encuentran en Costa-Lima y Suslick (2006) y en Brandão, Dyer y Hahn (2012). Este tratamiento analítico de la volatilidad presenta fuertes limitaciones, especialmente, cuando se identifican múltiples fuentes de incertidumbre.

${ }^{6}$ Bajo el enfoque MAD, Copeland y Antikarov (2001) proponen tratar el valor del proyecto sin opciones como si se tratara de un activo negociado, es decir, que asumen el VPN estático como el mejor estimador del valor de mercado del proyecto. 
(2005) resaltan las ventajas en la implementación del modelo binomial, dada la facilidad para el cálculo de los parámetros, así como su presentación más simple e intuitiva. Además, el árbol binomial facilita el mapeo de todas las acciones alternativas viables que dependen de los futuros posibles estados de naturaleza y, con ello, el proceso de toma de decisión de inversión.

Sin embargo, y a pesar de las ventajas del modelo binomial, se requiere de un modelo de valoración mucho más robusto que permita contemplar un tratamiento adecuado para las múltiples fuentes de incertidumbre. En este campo se identifican los desarrollos de Boyle, Evnine y Gibbs (1989), Kamrad y Ritchken (1991), Herath y Kumar (2006), así como propuestas alternativas de Gamba y Trigeorgis (2007) y Korn y Muller (2009), modelos en los cuales se incorporan múltiples procesos estocásticos (correlacionados o no correlacionados), así como probabilidades condicionales, para aproximar la dinámica propia de cada incertidumbre.

El propósito de este trabajo es presentar los diferentes modelos que se han desarrollado para tratar la presencia de múltiples incertidumbres en la valoración de opciones reales. Para ello, el documento está organizado como sigue. En la sección 2 se presenta de forma general el modelo binomial y sus características. Luego, en la sección 3 se presentan de forma general los diferentes modelos $k$-dimensionales y se resaltan sus ventajas y desventajas en cada caso. La sección 4 presenta una aplicación sencilla donde se valora una opción Rainbow (con múltiples fuentes de incertidumbre). Finalmente, se presentan las conclusiones del trabajo.

\section{El modelo binomial}

Para iniciar, se asume que el precio del activo subyacente $S_{(t \in[0, T])}$ sigue un proceso binomial multiplicativo para un periodo de tiempo discreto, definido en el espacio de probabilidad $(\Omega, F, P)$ con $\Omega=\left(\omega_{1}, \omega_{2}\right)$, donde el precio del activo subyacente en el futuro $(T=1)$ solo puede subir (estado $\left.\omega_{1}\right)$ o caer (estado $\left.\omega_{2}\right)$. Si se tiene un mercado financiero (simple) con dos fechas: $t=0$ (hoy) y $T$ (el vencimiento), por ejemplo, dentro de un año, y tres activos financieros: i) un bono, ii) una acción y iii) un derivado financiero (por ejemplo, una opción call), entonces, el precio de la acción en $t=0$ es conocido e igual a $S_{0}$, pero su precio en $T$, es decir, $S_{1}$ es incierto. Esta incertidumbre se modela estocásticamente al definir el precio en los dos estados de naturaleza ${ }^{7}$, donde el precio puede subir a $S_{1}^{u}$ en el

${ }^{7}$ La incertidumbre representa el hecho de que no se sabe con certeza cuál de los dos estados de naturaleza ocurrirá en $T$, cuando cada uno tiene cierta probabilidad de ocurrir. 
estado al alza o de crecimiento con una probabilidad $p_{u}$ o caer a $S_{1}^{d}$ en el estado a la baja o de decrecimiento con una probabilidad $p_{d}$ (ver figura 1$)$.

Figura 1: Árbol binomial del activo subyacente

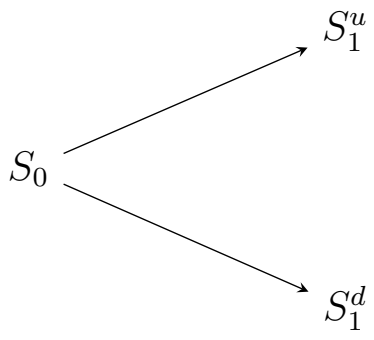

Fuente: elaboración propia.

Los precios $S_{1}^{u}$ y $S_{1}^{d}$ pueden verse como los resultados de multiplicar $S_{0}$ por dos factores: $u$ y $d$, es decir $S_{1}^{u}=S_{0} \times u$ y $S_{1}^{d}=S_{0} \times d$. Cabe resaltar que los parámetros $u$ y $d$ dependen de la volatilidad del activo subyacente, si esta se conoce, entonces se puede modelar el precio para el momento $T$. Para ello, se tiene que $u=e^{\sigma \sqrt{T}} \mathrm{y}$ $d=e^{-\sigma \sqrt{T}}$.

Por su parte, el precio del bono en $t=0$ está dado por $B_{0}=1$, entonces en $T$ este es $B_{T}=e^{r \times T}$. Ahora, si se tiene una opción call cuyo valor en $T$ es una función del precio del activo subyacente y está determinado por $C_{T}=\max \left(S_{T}-K, 0\right)$, donde $K$ es el precio de ejercicio. Entonces para $C_{T}$ se tiene

$$
\begin{aligned}
& C_{u}=\max \left(S_{T}^{u}-K, 0\right) \\
& C_{d}=\max \left(S_{T}^{d}-K, 0\right)
\end{aligned}
$$

Conocidos $C_{u}$ y $C_{d}$, el objetivo es determinar el valor de la opción, libre de oportunidades de arbitraje, en $t=0\left(C_{0}\right)$. La condición necesaria para demostrar la ausencia de oportunidades de arbitraje en el modelo es: $0<d<e^{r \times T}<u^{8}$, donde $r$ es la tasa libre de riesgo. Esto es equivalente a decir que $e^{r \times T}$ es una combinación convexa de los factores $u$ y $d$. Dado que $u$ ocurre con probabilidad $q_{u}$, y $d$ ocurre con probabilidad $q_{d}=1-q_{u}$, tenemos que

$$
e^{r \times T}=u q_{u}+d q_{d}
$$

${ }^{8} \mathrm{Si}$ la condición impuesta no se cumple se presentan oportunidades de arbitraje; por ejemplo, es posible obtener ganancias de arbitraje en un periodo de tiempo determinado si $e^{r T}<d$ al comprar acciones o $e^{r \times T}>u$ al realizar una venta en corto. 
donde: $q_{u}$ y $q_{d}$ son interpretadas como probabilidades riesgo-neutral (neutrales al riesgo), o de forma equivalente como la medida de probabilidad $Q$ o medida martingala equivalente (MME) y están determinadas por

$$
\begin{aligned}
& q_{u}=\frac{e^{r \times T}-d}{u-d} \\
& q_{d}=\frac{u-e^{r \times T}}{u-d}
\end{aligned}
$$

De manera que $q_{u}+q_{d}=1$. Como resultado, la ecuación (6) genera un valor único para la opción call en $t=0$

$$
\begin{array}{r}
C_{0}=e^{-r \times T} E^{Q}\left[\max \left(S_{1}-K, 0\right)\right] \\
C_{0}=e^{-r \times T}\left[q_{u} C_{u}+q_{d} C_{d}\right]
\end{array}
$$

Una ventaja de este modelo es que puede extenderse al contexto multiperiodo. Si se tiene que el vencimiento de la opción $(T)$ se divide en $n$ intervalos de tiempo con tamaño $h=\Delta t$, donde $h$ representa el paso discreto de un intervalo de tiempo $t$ a otro, para todo $t \in[0, T]$.

Ahora, si $u_{n}$ y $d_{n}$ representan los factores multiplicativos de crecimiento y decrecimiento del precio del subyacente para cada $h$, definidos en un espacio de probabilidad finito $(\Omega, \mathcal{F}, P)$, con $\Omega=\left(\omega_{1}, \omega_{2}\right)$, entonces el precio en un momento $h+1$ estará dado por $S_{(h t+1)}^{n}=u_{n} S_{h}^{n}$ y $S_{(h+1)}^{n}=d_{n} S_{h}^{n}$. Una característica importante del modelo es que los factores multiplicativos $u$ y $d$ son iguales para cada $h$, es decir, el árbol binomial mantiene la propiedad de recombinación, la cual se cumple siempre y cuando la volatilidad del activo subyacente permanezca constante para todo el periodo de valoración y, por tanto, las probabilidades riesgo-neutral $\left(q_{u}\right.$ y $\left.q_{d}\right)$ no cambian. Este enfoque representa el modelo 1-dimensional y permite resolver el problema de valoración de las opciones ${ }^{9}$ de forma sencilla y directa, al incorporar la dinámica del activo subyacente caracterizada por el parámetro de volatilidad en un proceso binomial multiplicativo.

Además, el modelo binomial ofrece una alternativa práctica y de fácil aplicación tanto en la valoración de opciones europeas como en las opciones americanas

\footnotetext{
${ }^{9}$ Sin embargo, el modelo CRR no es el único modelo que ha sido desarrollado en tiempo discreto. Se encuentran otros modelos alternativos que permiten estimar los parámetros como aproximación a la solución del modelo BSM, aunque bajo los mismos principios del CRR. Aquí se encuentran las propuestas de Rendleman y Bartter (1979), Hull y White (1988), Trigeorgis (1991), entre otros.
} 
(Copeland y Tufano, 2004). De igual forma, como indican Copeland y Antikarov (2001), el modelo binomial se puede ajustar fácilmente para reflejar cambios en la volatilidad, los puntos (óptimos) de decisión temprana, las decisiones secuenciales múltiples a través de opciones compuestas, o incluso múltiples fuentes de incertidumbre (opciones arcoíris), entre otras. Todo esto sin duda, muestra una ventaja notable frente al modelo BSM.

Sin embargo, las opciones reales representan decisiones estratégicas para hacer frente a las incertidumbres futuras, por tanto, el efecto conjunto de todas ellas debe ser considerado. No siempre, la representación de todas las fuentes de incertidumbre en un solo parámetro de volatilidad es adecuada. Es aquí donde surge el enfoque $k$-dimensional (o multinomial) para abordar esta limitación.

\section{Modelos $k$-dimensionales}

El modelo CRR puede representar la dinámica del activo subyacente durante el periodo de tiempo considerado $(T)$, sin embargo, este modelo presenta algunas limitaciones. Por ejemplo, si la opción cuenta con varios subyacentes, el modelo no se puede aplicar de forma directa. En el contexto de las opciones reales, este ámbito tiene aplicación cuando el proyecto de inversión cuenta con muchas incertidumbres. Esta limitación llevó al desarrollo de los modelos $k$-dimensionales. Por ejemplo, además de la incertidumbre de mercado ${ }^{10}$, el proyecto puede enfrentarse a la incertidumbre tecnológica (Copeland y Antikarov, 2001). En este caso, ambas fuentes de incertidumbre requieren un tratamiento adecuado dentro del modelo de valoración.

Lo anterior implica que el modelo de valoración debe mejorarse con el propósito de tomar decisiones de inversión correctas. Particularmente, el modelo CRR puede extenderse a un contexto $k$-dimensional. Si cada fuente de incertidumbre sigue un proceso MGB tal que

$$
d S_{i} / S_{i}=\mu_{i} d t+\sigma_{i} d W_{i} ; i=1, \ldots, k
$$

donde: $S_{i}$ representa la $i$-ésima fuente de incertidumbre, $\mu_{i}$ y $\sigma_{i}$ corresponden a la tasa de crecimiento instantánea y volatilidad de cada una, y $d W$ representa un proceso estándar de Wiener, donde $d W_{i}$ y $d W_{j}$ están correlacionados $E\left[d W_{i} d W_{j}\right]=$

${ }^{10}$ Esta incertidumbre puede estar representada, por ejemplo, en el precio del producto final (Brennan y Schwartz, 1985; Paddock et al., 1988; Cortázar y Schwartz, 1993) o en los costos operacionales (Pindyck, 1993), entre otros. 
$\rho_{i j} d t$. De esta forma, los métodos utilizados tratan de aproximar la distribución lognormal bivariada a una distribución discreta generalizada al contexto $k$-dimensional. En este campo se identifican, principalmente, los modelos propuestos por Boyle, Evnine y Gibbs (1989), Kamrad y Ritchken (1991), Herath y Kumar (2006) ${ }^{11}$, los cuales se presentan a continuación.

\subsection{Modelo de Boyle, Evnine y Gibbs (1989)}

La primera propuesta de valoración $k$-dimensional se identifica en Boyle, Evnine y Gibbs (1989), y es conocida como modelo BEG. El modelo BEG surge como una extensión del trabajo de Boyle (1988), quien introduce el modelo de valoración de opciones cuando hay dos variables de estado subyacentes (modelo bi-dimensional), al generalizar para $k$ variables de estado.

Boyle (1988) desarrolló una idea básica para valorar cualquier reclamación contingente cuyo payoff es una función lineal por partes de dos variables de estado subyacentes, siempre que estas tengan una distribución log-normal bivariada. Bajo esta lógica, construye un árbol bi-dimensional con cinco saltos (ramas) que representan la dinámica de los dos activos subyacentes, acompañado de sus respectivas probabilidades riesgo-neutral. Esta idea es generalizada en el modelo BEG, donde se asume que la distribución conjunta del proceso discreto $k$-dimensional cuenta con las propiedades del esquema de discretización del modelo CRR y, por tanto, los momentos de la distribución discreta coinciden con la distribución continua ${ }^{12}$. Una primera aproximación a esta propuesta se presenta inicialmente para 2 activos, al igual que Boyle (1988) y luego se generaliza para $k$ activos.

Para dos activos subyacentes, cuyos precios vienen dados por $S_{i}$ (ecuación 7), donde $i=1,2$, se encuentra que en el momento de tiempo futuro $T$ sus resultados al alza (crecimiento) y a la baja (decrecimiento) están determinados por $S_{i, 0} \times u_{i}$ y $S_{i, 0} \times d_{i}$, respectivamente. Si el periodo analizado se divide en $n$ intervalos (o pasos)

11 Además de estos se encuentran otros modelos alternativos propuestos por Nelson y Ramaswamy (1990), Ekvall (1996), este último conocido como modelo NEK, que buscan mejorar la convergencia y superar el problema de probabilidades negativas. También, se encuentran las propuestas de Korn y Muller (2009), Gamba y Trigeorgis (2007) que serán presentadas más adelante.

${ }^{12}$ La dinámica de un proceso de tiempo discreto converge con el proceso en tiempo continuo, lo que aumenta el número de pasos o intervalos de tiempo $(\Delta t)$, al igual que su función de distribución. La convergencia en la función de distribución es una condición suficiente para aproximar la convergencia del valor de la opción estimado a su valor verdadero, siempre que el payoff de la opción sea una función limitada de los precios de los activos subyacentes. 
de tamaño $\Delta t$ y su coeficiente de correlación es $\rho_{i, j}$, su función de distribución continua es aproximada por un proceso de 4 saltos (tabla 1), donde al igual que el modelo CRR: $u_{i}=e^{\sigma_{i} \sqrt{h}} \mathrm{y} d_{i}=e^{-\sigma_{i} \sqrt{h}}$.

Tabla 1: Aproximación de la distribución continua

\begin{tabular}{ccc}
\hline Salto & Probabilidad & Precios del activo \\
\hline$u_{1}, u_{2}$ & $p_{1}=p_{u u}$ & $S_{1} u_{1}, S_{2} u_{2}$ \\
$u_{1}, d_{2}$ & $p_{2}=p_{u d}$ & $S_{1} u_{1}, S_{2} d_{2}$ \\
$d_{1}, u_{2}$ & $p_{3}=p_{d u}$ & $S_{1} d_{1}, S_{2} u_{2}$ \\
$d_{1}, d_{2}$ & $p_{4}=p_{d d}$ & $S_{1} d_{1}, S_{2} d_{2}$ \\
\hline
\end{tabular}

Fuente: adaptado de Boyle et al. (1989).

Ahora, las probabilidades se determinan para los cuatro saltos de la distribución discreta, los cuales convergen a la distribución continua cuando $h$ tiende a cero (0). Para ello, se utiliza la función generadora de momentos. Sin embargo, su implementación requiere la transformación de la distribución log-normal de $S_{1}$ y $S_{2}$ a distribuciones normales. Si $S_{i, 0}$ representa el precio actual $(t=0)$ de los dos activos y $S_{i, 1}$, los cuatro posibles valores de cada uno $t=1$, se tiene

$$
R_{i}=\ln \left(\frac{S_{i, 1}}{S_{i, 0}}\right) ; i=1,2
$$

Entonces, la función característica es

$$
\psi_{h}\left(\theta_{1}, \theta_{2}\right)=E\left[e^{\left(i \theta_{1} R_{1}+i \theta_{2} R_{2}\right)}\right]
$$

Así, para los cuatro saltos se tiene

$$
\begin{array}{r}
\psi_{h}\left(\theta_{1}, \theta_{2}\right)=p_{1} E\left[e^{\left(i \sqrt{h}\left(\theta_{1} \sigma_{1}+\theta_{2} \sigma_{2}\right)\right.}\right]+p_{2} E\left[e^{\left(i \sqrt{h}\left(\theta_{1} \sigma_{1}-\theta_{2} \sigma_{2}\right)\right.}\right] \\
+p_{3} E\left[e^{\left(i \sqrt{h}\left(-\theta_{1} \sigma_{1}+\theta_{2} \sigma_{2}\right)\right.}\right]+p_{4} E\left[e^{\left(i \sqrt{h}\left(-\theta_{1} \sigma_{1}-\theta_{2} \sigma_{2}\right)\right.}\right]
\end{array}
$$

Al usar la expansión de Taylor e igualar la distribución discreta con su distribución continua bivariada, Boyle et al. (1989) encuentran que las probabilidades vienen determinadas por

$$
\begin{aligned}
& p_{1}=\frac{1}{4}\left(1+\rho+\sqrt{h}\left(\frac{\mu_{1}}{\sigma_{1}}+\frac{\mu_{2}}{\sigma_{2}}\right)\right) \\
& p_{2}=\frac{1}{4}\left(1-\rho+\sqrt{h}\left(\frac{\mu_{1}}{\sigma_{1}}-\frac{\mu_{2}}{\sigma_{2}}\right)\right)
\end{aligned}
$$




$$
\begin{aligned}
& p_{3}=\frac{1}{4}\left(1-\rho+\sqrt{h}\left(-\frac{\mu_{1}}{\sigma_{1}}+\frac{\mu_{2}}{\sigma_{2}}\right)\right) \\
& p_{4}=\frac{1}{4}\left(1+\rho+\sqrt{h}\left(-\frac{\mu_{1}}{\sigma_{1}}-\frac{\mu_{2}}{\sigma_{2}}\right)\right)
\end{aligned}
$$

donde, $\sum_{i=1}^{m}=1$ y $m=2^{n}$ es el número de los distintos estados en cada $h$.

Por su parte, en su generalización para $k$ activos subyacentes, la solución viene determinada por

$$
p_{m}=\frac{1}{2^{k}}\left(\sqrt{h} \sum_{i=1}^{k} x_{i m}\left(\frac{\mu_{i}}{\sigma_{i}}\right)+\sum_{i=1}^{k-1} \sum_{j=1+1}^{k} x_{i j}^{m}\left(\rho_{i, j}\right)\right)
$$

Para $m=1,2, . ., 2^{k}, k \geqslant 2, \mathrm{y}$

$$
x_{i m}=\left\{\begin{array}{rc}
1 & \text { si el activo } i \text { tiene un salto al alza en el estado } m \\
-1 & \text { si el activo } i \text { tiene un salto a la baja en el estado } m
\end{array}\right.
$$

$x_{i j}^{m}=\left\{\begin{array}{rc}1 & \text { si los activos } i \text { y } j \text { tienen un salto en la misma dirección } \\ -1 & \text { si los activos } i \text { y } j \text { tienen un salto en direcciones opuestas }\end{array}\right.$

Este modelo representa la primera extensión del modelo CRR a múltiples subyacentes. Sin embargo, como indican Kamrad y Ritchken (1991) y Gamba y Trigeorgis (2007), este modelo presenta algunas características no deseables, como la posibilidad de obtener probabilidades negativas y presenta una convergencia lenta, lo que lo convierte en un enfoque de aplicación poco eficiente.

\subsection{Modelo de Kamrad y Ritchken (1991)}

El modelo propuesto por Kamrad y Ritchken (1991) (en adelante modelo KR), extiende el trabajo previo de Boyle et al. (1989) usando los mismos principios, pero incorpora un salto horizontal, el cual transforma el modelo binomial subyacente en un modelo trinomial. En su extensión, el salto horizontal se obtiene agregando una constante $\lambda$ en la fórmula del tamaño del salto, es decir, $u_{i}=e^{\sigma_{i} \sqrt{h}}$ se convierte ahora en $u_{i}=e^{\lambda_{i} \sigma_{i} \sqrt{h}}$.

Como resultado, el modelo KR agrega una probabilidad adicional al conjunto de probabilidades del modelo BEG, la cual representa la probabilidad de que no haya movimiento alguno $\left(p_{0}\right)$. Si $\lambda=1$, el modelo KR genera un resultado equivalente al modelo BEG y, por tanto, $p_{0}=0$. 
La generalización del modelo KR a $k$ activos subyacentes viene dada por

$$
P_{m}=\frac{1}{2^{k}}\left[\frac{1}{\lambda^{2}}+\frac{\sqrt{h}}{\lambda} \sum_{i=1}^{k} x_{i m}\left(\frac{\theta_{i}}{\lambda_{i}}\right)+\frac{1}{\lambda^{2}} \sum_{i=1}^{k-1} \sum_{j=1+1}^{k} x_{i j}^{m} \frac{\rho_{i, j}+\theta_{i} \theta_{j} \Delta t}{\lambda_{i} \lambda_{j}}\right]
$$

Para $m=1,2, . ., 2^{k} ; k \geqslant 2$ y $\lambda \geqslant 1$. Además, $\theta_{i}=\mu_{i} / \sigma_{i}$ y $p_{0}=1-\frac{1}{\lambda^{2}}$. De esta forma, el número total de probabilidades viene determinado por

$$
\sum_{m=1}^{2^{k}+1} p_{m}=1
$$

Un aspecto importante del modelo KR es el valor del parámetro $\lambda$, lo cual puede representar una dificultad en su aplicación, dado que los autores no proveen propuesta alguna de su estimación, es decir, este se determina de forma arbitraria. Esto genera dificultades y sesgos en su implementación. Por ejemplo, Herath y Kumar (2006) muestran que un $\lambda$ superior a 1,5 genera valores de la opción significativamente diferentes de la solución analítica. Además, ellos encuentran que el modelo KR ignora los términos de orden superior del paso de tiempo $h$ al aproximar el cálculo de las probabilidades y, en algunos casos, encuentran resultados negativos.

\subsection{Modelo de Herath y Kumar (2006)}

Para corregir el problema anterior, Herath y Kumar (2006) proponen límites para el parámetro de $\lambda$ y el coeficiente de correlación $\rho$. Este ajuste se identificará como modelo HK. Los autores proporcionan intervalos donde las probabilidades generadas siempre serán factibles y definen un método de estimación de $\lambda$. Este ajuste implica que ahora las probabilidades para $k$ activos subyacentes vienen dadas por

$$
P_{m}=\frac{1}{2^{k}}\left[\frac{1+\theta_{1}^{2} \Delta t}{\lambda_{1}^{2}}+\sqrt{\Delta t} \sum_{i=1}^{k} x_{i m}\left(\frac{\theta_{i}}{\lambda_{i}}\right)+\sum_{i=1}^{k-1} \sum_{j=1+1}^{k} x_{i j}^{m} \frac{\rho_{i, j}+\theta_{i} \theta_{j} \Delta t}{\lambda_{i} \lambda_{j}}\right]
$$

Para $m=1,2, . ., 2^{k}, k \geqslant 2, \theta_{i}=\mu_{i} / \sigma_{i}, \mathrm{y}$

$$
\begin{gathered}
p_{0}=1-\left(\frac{1+\theta_{i}^{2} \Delta t}{\lambda_{i}^{2}}\right) \\
\lambda_{i}=\sqrt{1+\theta_{i}^{2} \Delta t}
\end{gathered}
$$




\section{Opciones reales con múltiples incertidumbres}

Las opciones que dependen de múltiples fuentes subyacentes de incertidumbre pueden agruparse en dos tipos: opciones Rainbow y opciones Basket. Una opción Rainbow se pueden definir como aquella cuyo payoff depende de más de un activo subyacente. Un ejemplo común de una opción Rainbow es la opción de intercambio de activos, donde un activo puede intercambiarse por otro. Por su parte, las opciones Basket, aunque son similares a las Rainbow al depender de múltiples subyacentes, su payoff se determina a partir de una ponderación de los activos subyacentes. En el contexto de los activos reales y proyectos de inversión, este tipo de opciones representa la flexibilidad de la inversión en diferentes contextos (espera, abandono, expansión, etc.) en presencia de múltiples fuentes subyacentes de incertidumbre. Por ejemplo, estas opciones ayudan a evaluar la respuesta que tiene la gerencia o dirección del proyecto de inversión a la incertidumbre futura en los precios del producto final, la cantidad demandada, los costos operativos, la tasa de cambio, entre otros. Aunque los dos tipos de opciones (Rainbow y Basket) son aplicables al contexto de las opciones reales, es más común encontrar el primer tipo.

Con el propósito de implementar los modelos multi-dimensionales presentados atrás, se propone un caso de aplicación hipotético en el que se implementa un procedimiento numérico para la valoración de una opción real Rainbow de diferir la inversión. Por simplicidad, este será analizado en el contexto bi-dimensional con incertidumbres correlacionadas. Inicialmente se desarrolla un ejercicio comparativo para los modelos BEG, KR y HK.

\subsection{Aplicación en el contexto bi-dimensional}

Se tiene un proyecto con valor presente de $\$ 145$ millones (VP $=\$ 145$ millones) con una inversión requerida de $\$ 125$ millones y dos fuentes de incertidumbre: el precio del producto final $(P)$ y la cantidad demandada $(Q)$. Se asume que ambas fuentes de incertidumbre siguen un proceso estocástico del tipo MGB y vienen dadas por: $d P / P=\mu_{P} d t+\sigma_{P} d W_{P}$ y $d Q / Q=\mu_{Q} d t+\sigma_{Q} d W_{Q}$, donde, $\mu_{P}=0,06$ y $\mu_{Q}=0,04$ corresponden a la tasa de crecimiento instantáneo del precio y la cantidad, respectivamente. $\sigma_{P}=0,16$ y $\sigma_{Q}=0,1$ representan sus volatilidades, mientras $d W$ representa un proceso estándar de Wiener. Además, se tiene que la correlación entre $P$ y $Q$ es $\rho_{P Q}=0,4$. Cabe resaltar que en el ejercicio de valoración riesgoneutral, el parámetro $\mu_{i}$ debe sustituirse por $r-0,5 \sigma_{i}$ en cada caso. A continuación, se presenta el cálculo de los cuatro parámetros. 
$u_{P}: 1,1735$

$d_{P}: 0,8521$
$u_{Q}: \quad 1,1052$

$d_{Q}: \quad 0,9048$

Estos parámetros, junto con las probabilidades riesgo-neutral $\left(p_{s}\right)$, de forma análoga al modelo binomial, deben ser tales que la distribución de probabilidad discreta converja con la distribución lognormal bivariada cuando el tamaño del intervalo de tiempo $h=\Delta t$ tiende a cero, entonces, la media, la varianza y la correlación de los modelos en tiempo discreto y continuo deben ser iguales. En presencia de dos fuentes de incertidumbre, para cada $h$ se tienen 4 nodos en el árbol binomial, es decir, se tiene un árbol cuatrinomial ${ }^{13}$. Esta misma dinámica se asocia al valor de la opción.

Así, para cada nodo del árbol, el valor del subyacente se determina como el producto de la combinación de parámetros $u$ y $d$ de cada variable, es decir, si se presenta el estado de crecimiento $(u)$ para $P$ y $Q$ en el primer intervalo de tiempo, $V_{u u}$ se determina como: $V_{u u}=V_{0} \times u_{P} \times u_{Q}$. Esta misma dinámica se aplica para los demás nodos del árbol. La figura 2 muestra la forma del árbol del valor subyacente con dos incertidumbres.

Figura 2: Árbol cuatrinomial del valor del proyecto

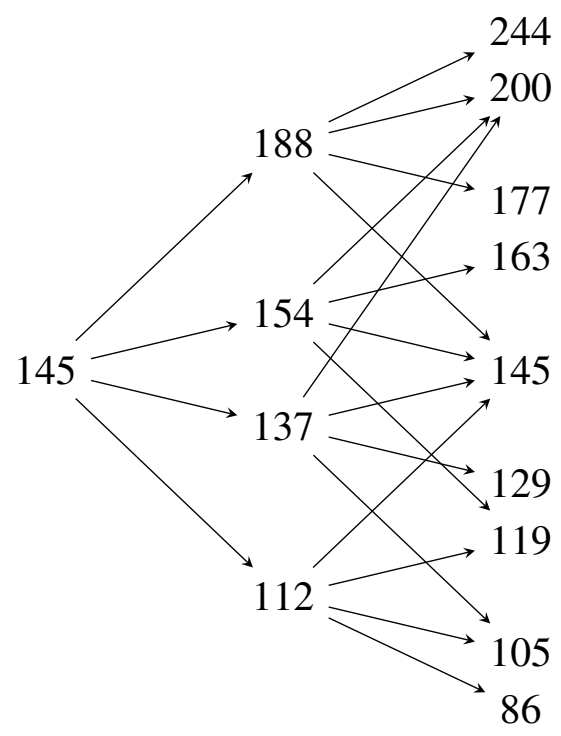

Fuente: elaboración propia.

\footnotetext{
${ }^{13}$ El modelo cuatrinomial es equivalente a uno binomial de dos variables. Este nuevo árbol tiene, para cada nodo, cuatro ramas, a diferencia de las dos ramas que presenta el árbol binomial.
} 


\subsection{Cálculo de las probabilidades riesgo-neutral}

El cálculo de las probabilidades para los modelos BEG, KR y HK se realiza aplicando las ecuaciones (10), (11), (12), (13). En el modelo KR se asume un parámetro de extensión $\lambda=1$, por tanto, sus resultados son equivalentes a los del modelo BEG. Además, para el modelo HK se presentan los parámetros $\theta_{P}=0,38$, $\theta_{Q}=0,40, \lambda_{P}=1,0680$ y $\lambda_{Q}=1,0770$. La tabla 2 resume los resultados.

Tabla 2: Probabilidades riesgo-neutral

\begin{tabular}{ccccc}
\hline Modelo & $P_{u u}$ & $P_{u d}$ & $P_{d u}$ & $P_{d d}$ \\
\hline BEG & 0,4394 & 0,1144 & 0,2606 & 0,1856 \\
KR & 0,4394 & 0,1144 & 0,2606 & 0,1856 \\
HK & 0,5502 & 0,1254 & 0,1889 & 0,1355 \\
\hline
\end{tabular}

Fuente: elaboración propia.

Además, se encuentra que para los modelos KR y HK, la probabilidad $p_{0}=0$.

\subsection{Determinación del valor de la opción}

Finalmente, se realiza la valoración de la opción Rainbow. Para ello, se define un periodo de análisis $(T)$ de 2 años y una tasa libre de riesgo del 3\%. El valor de la opción para cada modelo es

Modelos BEK y KR: $\quad \$ 46,2$

Modelo HK: $\quad \$ 60$

Cabe resaltar que el valor de la opción que proveen los modelos BEK y KR coincide dado que $\lambda=1$. Un mayor valor de este parámetro, por ejemplo, un $\lambda=1,1$ no solo originaría un valor de la opción diferente en este caso, sino que ahora incorporaría la probabilidad $p_{0}$, ahora con valor $p_{0}=0,1736$, lo que genera un árbol pentanomial. Nuevamente, la dificultad radica en cómo determinar este parámetro. 


\section{Modelos alternativos}

\subsection{Modelo de Korn y Muller (2009)}

Korn y Muller (2009) desarrollaron un modelo alternativo que busca superar sus limitaciones en la modelación del proceso $k$-dimensional. Este modelo, conocido como el enfoque de árboles ortogonales (en adelante modelo KM), se basa en el método general de transformación Decoupling Approach del modelo BSM para múltiples subyacentes, y usa los métodos de descomposición espectral y de Cholesky. Para ello, siguen la propuesta de Hull y White $(1990)^{14}$ para separar el efecto del parámetro de correlación en las probabilidades de transición, evitando así la presencia de probabilidades negativas, además de que el proceso simplifica la construcción del árbol $k$-dimensional.

Si la dinámica del precio de $k$ activos viene dada por $X_{t}=\ln \left(S_{t}\right)$, donde $d X_{i}=$ $\mu_{i} d t+\sigma_{i} d W_{t}^{i}$ con $i=1,2$ y $\mu_{i}=r-0.5 \sigma_{i}$, mientras la matriz de varianzas y covarianzas se representa como

$$
\Sigma=\left(\begin{array}{ccc}
\sigma_{1}^{2} & \ldots & \rho_{1 k} \sigma_{1} \sigma_{k} \\
\vdots & \ddots & \vdots \\
\rho_{1 k} \sigma_{1} \sigma_{k} & \cdots & \sigma_{k}^{2}
\end{array}\right)=G D G^{T}
$$

donde: $G$ es la matriz $k \times k$ y $D$ es la diagonal ${ }^{15}$. Además, se asume que $\Sigma$ es semidefinida-positiva, $G$ y $D$ son invertibles y los elementos de $D$ son diferentes de cero. La descomposición elemental se puede expresar como

$$
\rho_{i j} \sigma_{i} \sigma_{j}=\sum_{h=1}^{k} g_{i j} g_{h h} g_{j h}
$$

${ }^{14}$ Hull y White (1990) proponen el siguiente procedimiento de transformación para un proceso 2-dimensional que caracteriza el precio de dos activos: $S=S_{1}, S_{2}$, con dinámica: $d S_{i}=S_{i}\left(\mu_{i} d t+\right.$ $\left.\sigma_{i} d W_{t}^{i}\right) ; i=1,2$. La transformación del proceso original $S$ a un nuevo proceso $\psi$ con componentes independientes está dada por:

$$
\psi=\left(\begin{array}{cc}
\sigma_{2} & \sigma_{1} \\
\sigma_{2} & -\sigma_{1}
\end{array}\right) S
$$

${ }^{15}$ Este resultado se obtiene al utilizar la descomposición espectral, donde cada elemento de $D$ es un valor propio de $\Sigma$. De forma alternativa, al usar la descomposición de Cholesky se llega a un resultado equivalente donde $\Sigma=G G^{T}$ y $G$ es una matriz triangular y cada elemento de $D$ es igual a 1. 
para $h=1, . ., k$. De esta forma, Korn y Muller (2009) muestran que la transformación $Z=G^{-1} X$ genera $k$ movimientos Brownianos independientes. Así, el proceso que sigue $Z$ está dado por

$$
d Z_{t}^{i}=\alpha_{i} d t+\sqrt{d_{i i}} d W_{t}^{i}
$$

para $i=1, . ., k$, donde: $Z_{0}=G^{-1} X_{0}, \alpha_{i}=G^{-1} \mu_{i}$. A partir de este resultado se discretiza el proceso. Para ello, proponen la siguiente configuración

$$
Z_{h}^{N}=\left(\begin{array}{c}
Z_{h-1,1}^{N}+\alpha_{1} \Delta t+q_{h, 1} \sqrt{d_{11}} \sqrt{\Delta t} \\
\ddots \\
Z_{h-1, k}^{N}+\alpha_{k} \Delta t+q_{h, k} \sqrt{d_{k k}} \sqrt{\Delta t}
\end{array}\right)
$$

donde:

$$
x_{i m}=\left\{\begin{aligned}
1 & \text { con probabilidad } 0,5 \\
-1 & \text { con probabilidad } 0,5
\end{aligned}\right.
$$

Finalmente, en la valoración de la opción se transforma el proceso $k$-dimensional discreto anterior para reflejarlos en términos del proceso de precio inicial. Esta transformación viene dada por

$$
Y_{N}^{N}=\left(e^{G_{1} Y}, \ldots, e^{G_{k} Y}\right)^{T}
$$

En la implementación del modelo, los autores proponen un algoritmo de aplicación del método que consta de:

1. Descomponer la matriz de varianzas-covarianzas en $G D G^{T}$ utilizando la descomposición espectral o de Cholesky.

2. Transformar el proceso $k$-dimensional de activos subyacentes $X$ en $Z$, de modo que el nuevo proceso $(Z)$ tenga movimientos Brownianos independientes.

3. Discretizar el proceso utilizando la expresión (16).

4. Construir el árbol $k$-dimensional con componentes independientes utilizando el proceso discreto definido en la ecuación (17).

5. Calcular el payoff en todos los nodos y utilizar el proceso de inducción hacia atrás.

De esta forma, los autores aproximan cada componente del proceso $Y_{N}^{N}$ en un proceso discreto, es decir, un árbol binomial 1-dimensional. 


\subsection{Modelo de Gamba y Trigeorgis (2007)}

Gamba y Trigeorgis (2007) proponen un modelo multi-dimensional para valorar opciones que dependen de múltiples subyacentes que siguen procesos del tipo MGB correlacionados, conocido como modelo generalizado log-transformado (GLT, por sus siglas en inglés). Este modelo, a diferencia de los anteriores, consiste en: i) la transformación logarítmica del proceso de precio de los activos subyacentes basado en el trabajo de Trigeorgis (1991) y, ii) un cambio de base para aproximar numéricamente una dinámica no correlacionada en los procesos del precio de los activos. Su propuesta no solo corrige la presencia de probabilidades negativas y el problema de dimensionalidad presente en algunos de los modelos anteriores, sino que mejora de forma significativa la eficiencia del algoritmo multidimensional.

Si se consideran $k$ activos, tales que $\left(S^{T}=\left(S_{1}, \ldots, S_{k}\right)\right)^{16}$ correlacionados, donde $S$ sigue un proceso estocástico $k$-dimensional del tipo MGB

$$
d S_{i} / S_{i}=\mu_{i} d t+\sigma_{i} d W_{i} ; i=1, \ldots, k
$$

donde los procesos estándar de Wiener para los activos $i$ y $j$ están correlacionados, de tal modo que $E\left[d W_{i} d W_{j}\right]=\rho_{i j} d t$ para todo $i \neq j$. Ahora, al tomar el logaritmo del valor del proceso $S, Y_{i}=\ln \left(S_{i} / s_{i}\right)$ y al aplicar el lema de Itô, se encuentra que la dinámica de $Y^{T}=\left(Y_{1}, \ldots, Y_{k}\right)$ viene dada por

$$
d Y=a d t+\sigma d W
$$

donde: $a^{T}=\left(a_{1}, \ldots, a_{k}\right)$ con $a_{i}=a_{i}-1 / 2 \sigma_{i}^{2}$ y $d W^{T}=\left(d W_{1}, \ldots, d W_{k}\right)$. Además, al aplicar las reglas de multiplicación del cálculo estocástico: $d t d W=0,(d t)^{2}=0$, $d W_{i} d W_{j}=\rho_{i j} d t$, se tiene que

$$
\Sigma=\left(\begin{array}{cccc}
1 & \rho_{12} & \ldots & \rho_{1 k} \\
\rho_{21} & 1 & \ldots & \rho_{1 k} \\
\vdots & \vdots & \ddots & \vdots \\
\rho_{k 1} & \rho_{k 2} & \ldots & 1
\end{array}\right) \quad \sigma=\left(\begin{array}{cccc}
\sigma_{1} & 0 & \ldots & 0 \\
0 & \sigma_{2} & \ldots & 0 \\
\vdots & \vdots & \ddots & \vdots \\
0 & 0 & \ldots & \sigma_{k}
\end{array}\right)
$$

Luego, mediante una transformación de la base (de los rendimientos de los activos) se crea una aproximación no correlacionada de su dinámica (y). Esta transformación implica que también se debe cambiar la base del payoff de la opción. Si

\footnotetext{
${ }^{16}$ Donde $\mathrm{T}$ denota la matriz transpuesta.
} 
$\Pi(Y)$ denota el payoff original, y $W$ representa la matriz que muestra el cambio de base, entonces el payoff ajustado con respecto a la nueva base es $\tilde{\Pi}(y)=\Pi(W y)$. Donde, $y=W^{T} Y$ representa el retorno de un portafolio sintético (replicante) construido a partir de una combinación lineal de activos disponibles en el mercado, y $y$ sigue un proceso de difusión $d y=A d t+B d W$, donde $A=W^{T}$ a y $B=W^{T} \sigma$. Además, la matriz de covarianzas de $d y$ es $d y d y^{T}=\wedge d t$. Esto significa que todos los elementos de $y^{T}$ son no correlacionados.

Si el valor esperado del payoff de la opción ( $(\hat{\Pi})$ riesgo-neutral, denotado como $\tilde{F}$, es igual al valor de la opción (П) en su vencimiento $T$, se tiene

$$
\tilde{F}(y(t))=e^{-r \times T} E_{y}[\tilde{\Pi}(y(T))]=e^{-r \times T} E_{Y}[\Pi(Y(T))]
$$

donde, $E_{y}[\cdot]$ denota el valor esperado riesgo-neutral de $y$, mientras $E_{Y}[\cdot]$ el valor esperado del proceso $Y$. Esta dinámica puede ser aproximada mediante un árbol binomial multidimensional adecuado.

Ahora, $\tilde{F}(y(t))$ puede ser aproximada a una distribución discreta (binomial), al dividir el vencimiento $T$ en $n$ intervalos de tamaño $\Delta t=T / n$. Esta aproximación del proceso $y$ se representa como $\hat{y}^{T}=\left(\hat{y}_{1}, \ldots, \hat{y}_{k}\right)$. Así, $\hat{y}^{n}$ representa el proceso aproximado en tiempo discreto con $n$ pasos. Este enfoque binomial mejorado para el caso bi-dimensional se representa como:

$$
W=\left(\begin{array}{cc}
\left(\frac{\lambda_{1}}{\sigma_{2} \sigma_{1}}-\frac{\sigma_{1}}{\sigma_{2}}\right) / \rho c_{1}\left(\frac{\lambda_{2}}{\sigma_{1} \sigma_{2}}-\frac{\sigma_{2}}{\sigma_{1}}\right) / \rho c_{2} & \\
1 / c_{1} & 1 / c_{2}
\end{array}\right)
$$

donde: $\Lambda=\left(\lambda_{i}\right), \mathrm{y}$

$$
\begin{gathered}
c_{i}=\sqrt{1+\frac{\left(\lambda_{i}-\sigma_{2}^{2}\right)^{2}}{\rho^{2} \sigma_{1}^{2} \sigma_{2}^{2}}} \\
\lambda_{1}=\frac{1}{2}\left(\sigma_{1}^{2}+\sigma_{2}^{2}-\sqrt{\sigma_{1}^{4}-2\left(1-2 \rho^{2}\right) \sigma_{1}^{2} \sigma_{2}^{2}+\sigma_{2}^{4}}\right) \\
\lambda_{2}=\frac{1}{2}\left(\sigma_{1}^{2}+\sigma_{2}^{2}+\sqrt{\sigma_{1}^{4}-2\left(1-2 \rho^{2}\right) \sigma_{1}^{2} \sigma_{2}^{2}+\sigma_{2}^{4}}\right)
\end{gathered}
$$

Entonces, los procesos del retorno del portafolio sintético son $d y_{i}=A_{i} d t+$ $B_{i 1} d W_{1}+B_{i 2} d W_{2}$, con $i=1,2$. Ahora, la aproximación discreta de $y$ en el intervalo $[0, T]$ con $n$ pasos, es $\hat{y}^{T}=\left(\hat{y}_{1}, \hat{y}_{2}\right)$ con dinámica

$$
\hat{y}_{i}(t)=\hat{y}_{i}(t-1)+\ell_{i} U_{i}(t) ; i=1,2 .
$$


donde: $U_{i}(t)$ representa un set de variables aleatorias bi-variadas, tales que

$$
\left(U_{1}, U_{2}\right)=\left\{\begin{aligned}
(1,1) & \text { con probabilidad } p_{1} \\
(1,-1) & \text { con probabilidad } p_{2} \\
(-1,1) & \text { con probabilidad } p_{3} \\
(-1,-1) & \text { con probabilidad } p_{4}
\end{aligned}\right.
$$

y $\sum_{i=1}^{k} p_{i}=1$. Además, los parámetros del proceso en tiempo discreto son

$$
\begin{gathered}
\kappa_{i}=A_{i} \Delta t \\
\ell_{i}=\sqrt{\lambda_{i} \Delta t+\kappa_{i}^{2}} \\
L_{i}=\kappa_{i} / \ell_{i}
\end{gathered}
$$

Por su parte, las probabilidades vienen determinadas por

$$
p_{s}=\frac{1}{4}\left(1+\delta_{12}(s) L_{1} L_{2}+\delta_{1}(s) L_{1}+\delta_{2}(s) L_{2}\right) ; s=1, \ldots, 4
$$

donde:

$$
\delta_{i}(s)=\left\{\begin{array}{rc}
1 & \text { si el valor del activo } i \text { sube }(u) \\
-1 & \text { si el valor del activo } i \text { cae }(d)
\end{array}\right.
$$

Con estos parámetros, la función característica del proceso en tiempo discreto coincide con la función característica del proceso en tiempo continuo para cualquier intervalo $\Delta t$, tal que

$$
\begin{gathered}
\ell_{i} \sum_{s=1}^{S} p(s) \delta_{i}(s)=A_{i} \Delta t \\
\ell_{i}^{2}-\left(A_{i} \Delta t\right)^{2}=\lambda_{i} \Delta t \\
\ell_{i} \ell_{j} \sum_{s=1}^{S} p(s) \delta_{1}(s) \delta_{2}(s)=0 ; i \neq j
\end{gathered}
$$

En la generalización de este resultado a un proceso $k$-dimensional, donde $\hat{y}^{T}=$ $\left(\hat{y}_{1}, \ldots, \hat{y}_{k}\right)$ y $U=\left(U_{1}, \ldots, U_{k}\right)$ es un set de $k$ variables aleatorias binomiales, se encuentra que las probabilidades vienen determinadas por

$$
p_{s}=\frac{1}{S}\left(1+\sum_{1 \leq i \leq j \leq K} \delta_{i j}(s) L_{i} L_{j}+\sum_{k=1}^{K} \delta_{i}(s) L_{i}\right) ; s=1, \ldots, S .
$$

Finalmente, dadas las ecuaciones (21), (22) y (23), se encuentra que el valor de la opción es

$$
\tilde{F}(y(t))=\max \left(\tilde{\Pi}(y(t)), e^{-r \times \Delta t} E_{y}[\tilde{F}(y(t+1))]\right)
$$

La ecuación (24) representa la versión discreta de la ecuación de Bellman. 


\section{Conclusiones}

El modelo de valoración de opciones en tiempo discreto (modelo binomial) provee un esquema de solución al problema de valoración basado en la aproximación del proceso estocástico utilizando la función binomial; de esta forma, el proceso se aproxima para cada momento $t$ tomando solo dos estados (sube o cae) con sus respectivas probabilidades (riesgo-neutral) asociadas. Este modelo representa una aplicación simple y directa y ha tenido gran acogida en el campo de valoración de opciones reales. Sin embargo, la presencia de múltiples fuentes de incertidumbre, como es característico en activos reales y proyectos de inversión, requiere de ajustes en el modelo de valoración.

En este contexto surgen los modelos multi-dimensionales. Bajo este esquema, el proceso estocástico $k$-dimensional se aproxima a un proceso discreto multivariado que conserva las mismas propiedades del modelo binomial, entre estas, la propiedad de recombinación, siempre y cuando el parámetro de volatilidad se asuma constante. Se debe tener presente que la presencia de un mayor número de incertidumbres incrementa el número de ramas para cada nodo del árbol, lo cual aumenta el problema de dimensionalidad. Desde esta perspectiva se encuentran los modelos BEG, KR y HK. Aunque estos modelos aportan resultados interesantes, presentan ciertas limitaciones que los vuelven indeseables en su aplicación, por ejemplo, la presencia de probabilidades negativas. Como alternativa, se encuentran los modelos desarrollados por Gamba y Trigeorgis (2007) y Korn y Muller (2009) que, aunque presentan un mayor nivel de complejidad numérica, corrigen este tipo de limitaciones.

\section{Referencias}

Amram, M. y Kulatilaka, N. (1999). Real options: Managing strategic investment in an uncertain world. Boston: Harvard Business School Press.

Black, F. y Scholes, M. (1973). The pricing of options and corporate liabilities. Journal of Political Economy, 81(3), 637-659.

Borison, A. (2005). Real options analysis: Where are the emperor's clothes? Journal of Applied Corporate Finance, 17(2), 17-31.

Boyle, P. (1988). A lattice framework for option pricing with two state variables. Journal of Financial and Quantitative Analysis, 23(1), 1-12. 
Boyle, P., Evnine, J. y Gibbs, S. (1989). Numerical evaluation of multivariate contingent claims. The Review of Financial Studies, 2(2) 241-250.

Brandão, L. Dyer, J., y Hahn, W. (2005). Using binomial decision trees to solve real options valuations problems. Journal of Decision Analysis, 2(2), 69-88.

Brandão, L., Dyer, J. y Hahn, W. (2012). Volatility estimation for stochastic project value models. European Journal of Operational Research, 220(3), 642-648.

Brennan, M. y Schwartz, E. (1985). Evaluating natural resource investments. Journal of Business, 58(2), 135-157.

Copeland, T. y Antikarov, V. (2001). Real Options: A Practitioner's Guide. New York: Texere Publishing Ltd.

Copeland, T. y Tufano, P. (2004). A real world way to manage real options. Havard Business Review, 1-12.

Cortázar, G. y Schwartz, E. (1993). A compound option model of production and intermediate inventories. Journal of Business, 66(4), 517-540.

Costa-Lima, G. y Suslick, S. (2006). Estimating the volatility of mining projects considering price and operating cost uncertainties. Resources Policy, 31(2), 86-94.

Cox, J., Ross, S. y Rubinstein, M. (1979). Option pricing: A simplified approach. Journal of Financial Economics, 7(3), 229-263.

Davis, G. (1998). Estimating volatility and dividend yield when valuing real options to invest or abandon. The Quarterly Review of Economics and Finance, 38(3), 725754.

Dixit, A. y Pindyck, R. (1994). Investment under Uncertainty. New Jersey: Princeton University Press.

Ekvall, N. (1996). A lattice approach for pricing of multivariate contingent claims. European Journal of Operational Research, 91(2), 214-228.

Gamba, A. y Trigeorgis, L. (2007). An improved binomial lattice method for multidimensional options. Applied Mathematical Finance, 14(5), 453-475.

Herath, H. y Kumar, P. (2006). Multinomial approximating models for options. Advances in Investment Analysis and Portfolio Management, (2), 199-226.

Hull, J. y White, A. (1988). The use of the control variate technique in option pricing. Journal of Financial and Quantitative analysis, 23(3), 237-251. 
Hull, J. y White, A. (1990). Valuing derivative securities using the explicit finite difference method. The Journal of Financial and Quantitative Analysis, 25(1), 87100 .

Kamrad, B. y Ritchken, P. (1991). Multinomial approximating model for options with k-state variables. Management Science, 37(12), 1640-1652.

Korn, R. y Müller, S. (2009). The decoupling approach to binomial pricing of multiasset options. The Journal of Computational Finance, 12(3), 1-30.

Merton, R. (1973). The theory of rational option pricing. The Bell Journal of Economics and Management Science, 4(1), 141-183.

Merton, R. (1977). On the pricing of contingent claims and the Modigliani-Miller theorem. Journal of Finance, 29(5), 241-249.

Myers, S. (1977). Determinants of corporate borrowing. Journal of Financial Economic, 5(1), 147-175.

Nelson, D. y Ramaswamy, K. (1990). Simple binomial processes as diffusion approximations in financial models. The Review of Financial Studies, 3(3), 393-430.

Paddock, J., Siegel, D., y Smith, J. (1988). Option valuation of claims on real assets: The case of offshore petroleum leases. The Quarterly Journal of Economics, 103(3), 479-508.

Pindyck, R. (1993). Investment of uncertain cost. Journal of Financial Economics, 34(1), 53-76.

Rendleman, R. y Bartter, B. (1979). Two-state option pricing. The Journal of Finance, 34(5), 1093-1110.

Smit, H. y Trigeorgis, L. (2004). Strategic investment: Real Options and Games. New Jersey: Princeton University Press.

Trigeorgis, L. (1991). A log-transformed binomial numerical analysis method for valuing complex multioption investments. Journal of Financial and Quantitative Analysis, 26(1), 309-326

Trigeorgis, L. (1996). Real Options: Managerial Flexibility and Strategy in Resource Allocation. Cambridge, Mass.: The MIT Press. 